\title{
Diskussion zum Vortrag von Thomas Simon
}

\author{
Leitung: Wolfgang Sellert
}

SELLERT:

Vielen Dank für dieses Referat, das mit der moralischen und intellektuellen Steuerung des Staates im 16. Jahrhundert begann. Es schlossen sich Gedanken zu der im 17. Jahrhundert bestehenden Vorstellung zur disciplina publica an. Ihnen folgten Ausführungen zur Kameralwissenschaft im 18. Jahrhundert, wo, wie Sie gezeigt haben, für den Erziehungsgedanken Fragen der Ökonomie und der Nützlichkeit eine Rolle gespielt haben. Ihre Ausführungen endeten mit Überlegungen zur liberalen Epoche des 19. Jahrhunderts, in der, wie Sie formuliert haben, der Erziehungsgedanke delegitimiert wurde. Was nun das Polizeirecht betrifft, so hätte man zwar etwas mehr über den Erziehungsgedanken in den Polizeiordnungen selbst erwartet. Offenbar findet sich dort aber nur Weniges dazu, sodass man - wie Sie - die sogenannte Regiments- oder die allgemeine politische Literatur befragen muss, um einschlägige Motive zum Erziehungsgedanken in den Polizeiordnungen zu finden. Ehe ich mich zu weiteren Punkten Ihres Referats äußere, möchte ich mich zunächst auf die Diskussionsleitung beschränken und darf zunächst Herrn Starck das Wort geben.

STARCK:

Ich möchte mich für diesen schönen Vortrag bedanken, der mit WELCKER angefangen und mit WelCKER aufgehört hat. Das war eine sehr schöne Komposition dieses Vortrages. Ich möchte zwei Fragen stellen. Die eine Frage knüpft an Ihre häufige Bemerkung an über das Verhältnis von Kirche und Staat. Sie haben sehr eindrucksvoll gezeigt, wie der Staat heute Aufgaben übernimmt, die die Kirche früher wahrgenommen hat. Hat das mit der Reformation zu tun? Die Frage könnte man beantworten, wenn man wüsste, wie die Entwicklung in Spanien oder in anderen Ländern, in denen es keine Reformation gab, verlief. Ist hier der Staat weiter liberaler oder desinteressierter an diesen Dingen geblieben? In Ländern oder in Kulturen, in denen es die Trennung von Staat und Kirche bzw. Religion nie gegeben hat, wie etwa in China oder auch in der byzantinischen Welt, hatte der Staat von vornherein eine erzieherische Aufgabe, weil die Kirche nur eine Art Behörde des Staates war oder die Religion nur in die Staatsaufgaben eingebunden war. Es gab in Deutschland im Gefolge der Schriften von Rousseau durchaus die Vorstellung, dass der Staat die Aufgabe der Erziehung hat. Karl Salomo Zachariae hat noch Anfang des 19. Jahrhunderts ein kleines 
Büchlein geschrieben über die Notwendigkeit der Erziehung durch den Staat. Das ist also die erste Frage.

Die zweite Frage knüpft an Frau Schumanns einleitende Bemerkung an: Wenn wir sagen, der liberale Staat erzieht nicht, dann muss man sehen, dass der liberale Staat vielleicht nicht so direkt erzieht wie wir das jetzt hier so vorgespielt bekommen haben, sondern der liberale Staat lässt den Bürger Konsequenzen seines Handelns tragen. Also: Wenn ich eine strafbare Handlung begehe, werde ich bestraft. Oder wenn ich einen Schaden erzeuge, gibt es einen Schadensersatzanspruch. Das weiß der Bürger, deswegen nimmt er sich zusammen. Wenn ein Schaden erzeugt worden ist, für den jemand verantwortlich ist, so muss dieser den Schaden ersetzen. Wenn das einmal durchgesetzt würde, dann würde man schon etwas mehr aufpassen, woher man - ich knüpfe an den Dioxinskandal an - sein Futtermittel bezieht und wer da alles mitgemischt hat. Das war eine zusätzliche Bemerkung, die nicht direkt zu Ihrem Vortrag ging, sondern mehr zu dem, was Frau Schumann gesagt hat.

SIMON:

Am Schluss haben Sie ja den entscheidenden Gesichtspunkt der „Erziehung“ angesprochen. Das war für mich auch die Frage bei den einführenden Worten von Frau Schumann: Kann man die moderne Gesetzgebung, die ein bestimmtes Verhalten sanktioniert, indem sie es mit einer nachteiligen Rechtsfolge belegt, oder die zu einem bestimmten Verhalten motivieren möchte, indem sie eine Belohnung, eine Subvention oder sonst irgendetwas verspricht, als erziehende Gesetzgebung bezeichnen? Meines Erachtens handelt es sich hier nicht um eine „Erziehung“, wie man sie in der Frühen Neuzeit beobachten kann. Denn der Anspruch auf das Innere, sozusagen auf die mentalen Strukturen des Menschen zuzugreifen, ist doch bei dieser modernen Gesetzgebung nicht in vergleichbarer Weise gegeben. Es ist eine andere Methode, aber eben auch der Anspruch ist schon ein anderer. Auch die moderne Gesetzgebung ist natürlich von bestimmten Steuerungsintentionen getragen. Die Gesellschaft soll in eine ganz bestimmte Richtung gelenkt werden. Aber man macht das mit Belohnung und Strafe und lässt die Frage, welche inneren Einstellungen der Mensch dazu entwickelt, vorsichtig ausgedrückt doch eher im Hintergrund stehen. Jedenfalls ist die Motivlage in keiner Weise vergleichbar mit derjenigen in der frühen Neuzeit, wo gerade die Kernidee darin bestand, den Menschen vor allem auch innerlich besser zu machen. Die moderne Gesetzgebung ist doch mehr auf das äußere Verhalten gerichtet. Sie ist darauf ausgerichtet, das äußerliche Verhalten zu steuern, und lässt die Frage der inneren Einstellung dazu dahingestellt. Die frühneuzeitliche Polizeigesetzgebung ist hingegen von dem Ziel motiviert, die mentalen Strukturen der Untertanen zu verändern. Bei dem reformationszeitlichen Politikverständnis 
kam es gerade darauf an, die Leute fromm zu machen, weil nur ein frommes Gemeinwesen der Gefahr der göttlichen Strafen entkommt.

$\mathrm{Zu}$ dem Verhältnis von Kirche und Staat bzw. zum Vergleich zwischen reformierten und katholischen Territorien kann sicher Herr Link mehr sagen. Aber von der Grundrichtung lassen sich doch zahlreiche Parallelen beobachten. Denn schon vor der Reformation entwickelt sich ja in deutschen Territorien das landesfürstliche Kirchenregiment. Natürlich bleibt dann die Autonomie des kirchlichen Bereiches in den katholischen Territorien stärker ausgeprägt als in den protestantischen Territorien, in denen die kirchlichen Institutionen in die staatlichen Verwaltungsstrukturen eingegliedert werden. In den katholischen Territorien behält der kirchliche Bereich eine größere Autonomie, wird aber auch einer straffen territorialstaatlichen Aufsicht unterstellt. Dahinter steht das politische Bestreben, kirchliche und polizeiliche Steuerung besser aufeinander abzustimmen. Das sind Politikgrundsätze, die gelten gleichermaßen für evangelische wie für katholische Territorien. Ich habe mich zwar nicht mit der spanischen Verwaltungsgeschichte beschäftigt, aber dieser Grundbefund, dass der Staat die Kirche zu kontrollieren und sich ihre Fähigkeit zur Wertevermittlung dienstbar zu machen beginnt, begegnet einem gleichermaßen in katholischen und evangelischen Territorien.

SELLERT:

Da mir zehn weitere Wortmeldungen vorliegen und wir die uns zur Verfügung stehenden Zeit nicht überschreiten sollen, werde ich jetzt drei Teilnehmern hintereinander das Wort erteilen und anschließend Herrn Simon Gelegenheit zur Erwiderung geben.

\section{EICHENHOFER:}

Sie sagten, dass die ökonomisch motivierte Erziehung im 18. Jahrhundert eingesetzt habe. Ich würde sagen, sie setzte schon viel früher ein, nämlich im 13./14. Jahrhundert, als die Bettelverbote erlassen wurden. Das ist für das Verständnis der Herausbildung einer Wirtschaftsgesellschaft von zentraler Bedeutung. Die Polizeiordnungen des ausgehenden Mittelalters und der frühen Neuzeit lebten von der Idee des Bettelverbotes und beförderten damit die Arbeitsgesellschaft als Lebensform. Man könnte fast so weit gehen zu sagen, der Frühliberalismus baut auf den Erziehungsleistungen der frühen Neuzeit auf, weil er die sich selbst reproduzierende Gesellschaft geschaffen hat. Und ein letzter Punkt: Vor dem Hintergrund dieser Bettelverbote entstand danach ein Wohlfahrtswesen, eine Leistungsverwaltung für diejenigen, die sich nicht selbst unterhalten können. Auch das geht bis in das 13./14. Jahrhundert zurück. 
SELLERT:

Herr Wißmann bitte.

WISSMANN:

Vielen Dank. Herr Simon, Sie haben ja sehr stark vom Gegensatz der frühen Neuzeit und des liberalen Rechtsstaates her argumentiert und ich möchte im Anschluss an Herrn Eichenhofer mehr die graduellen Übergänge pointieren. Zunächst einmal scheint mir einer deutlichen Unterstreichung wert zu sein, dass und warum „Erziehung durch Gesetz“ in der frühen Neuzeit in neuer Mächtigkeit einsetzt. Das hat einmal eine formelle Seite, das hatten Sie ja gesagt, das Gesetz wird als Herrschaftsmittel jetzt überhaupt erst nutzbar, zuvor gab es Herrschaft vom Sattel aus, aber eben nicht in der rationalen Form abstrakter Ordnung. Und es kommt sozusagen der historische Zufall der Reformation und Gegenreformation hinzu, der in dieser Konkurrenzsituation des Konfessionalismus auf einmal auch eine materielle Begründung von Herrschaftsansprüchen in neuer Weise notwendig macht. Es geht zu diesem Zeitpunkt aber gerade noch nicht darum, die Herrschaft als solche zu rechtfertigen, einen Bürgerstatus abzugrenzen von der Herrschaft, sondern es geht letztlich um Rationalität von Herrschaft, so habe ich Sie auch im Wesentlichen verstanden. Mein Einwand, meine Rückfrage setzt ein, wenn Sie darin einen klaren Gegensatz zum 19. Jahrhundert konstruieren. Ich würde als Gegenthese formulieren: Erst der liberale Rechtsstaat des 19. Jahrhunderts schafft sich wirksame Erziehungsinstitutionen und bedarf deshalb des allgemeinen erziehenden Gesetzes jenseits solcher Institutionen nicht mehr. Bis ins 19. Jahrhundert betreibt der Staat Erziehungseinrichtungen nur virtuell. Es gibt im Grunde keine Schule auf dem Land. Wenn man sich die Statistiken ansieht, gibt es zwar Schulordnungen, aber die Kinder gehen nicht in die Schule. Erst im 19. Jahrhundert wird das anders: Noch zu Beginn des 19. Jahrhunderts liegt die Quote, wenn man Preußen anschaut, bei 15-20\%, am Ende des 19. Jahrhunderts bei $90 \%$. Das heißt, hier wird auf einmal in staatlichen Institutionen erzogen und mir erscheint, Sie sind da ein wenig sozusagen der liberalen Ideologie auf den Leim gegangen.

SimON:

Aber das Thema hieß Erziehung in der Gesetzgebung, nicht generell in den Staatstätigkeiten. Das ist wesentlich.

WISSMANN:

Genau, aber es gibt sozusagen für das ganze Bild eben auch den Blick auf die Institutionen und je mehr ich wirksame Staatsinstitutionen habe, desto mehr kann ich die Erziehung durch allgemeine Gesetze zurücknehmen. Erlauben Sie mir noch eine Bitte um Ergänzung: Vielleicht könnten Sie uns noch etwas mehr zu 
der Art der Gesetze in der Frühen Neuzeit schildern? Das ist doch, wenn ich das richtig sehe, bis zum Preußischen Allgemeinen Landrecht eben auch ein anderes Gesetz als wir es heute kennen, nämlich ein bildmächtiges Gesetz, das mit Einzelbestimmungen arbeitet, mit einzelnen Verbotstatbeständen, die in langer Reihe gefasst werden. Was hat das für den Erziehungsanspruch für Konsequenzen?

SELLERT:

Herr Stolleis bitte.

STOLleis:

Nur eine ganz kurze Bemerkung. Zur Frage von Herrn Sellert, ob man den Gedanken anhand der Literatur oder anhand der Polizeiordnungen entwickelt, meine ich, man kann beides tun. Aus unserer gemeinsamen Arbeit an den Polizeiordnungen ergibt sich, dass sowohl das sittliche als auch das bessernde edukatorische Element in großen Massen in den Polizeiordnungen enthalten ist. Es bildet sogar den größten Anteil in dem frühen Block des 16. Jahrhunderts, verschiebt sich dann im 17. und 18. Jahrhundert etwas. Das Material ist überreich. Wir schätzen, dass es etwa 1 Mio. Gesetzgebungsakte dieser Art gibt. Die Register unserer Bände zeigen, welch enormes Material da bereitliegt. Also beide Wege, der theoriegeschichtliche und der dem Material folgende rechtsgeschichtliche, wären möglich gewesen. Herr Simon hat nun den Weg über die Literatur gewählt und dies ist völlig legitim.

Die zweite Bemerkung, im Anschluss an Herrn Eichenhofer: Ich gebe Ihnen völlig Recht in Bezug auf das frühe Einsetzen mit den Bettelverboten, auch mit den Hochzeits- und Feiergesetzen sowie mit den Verboten, zu viel zu konsumieren, aber trotzdem ist die eigentliche Verdichtung dieser Gesetzgebung erst seit dem 16. Jahrhundert festzustellen. Wir können das auch statistisch sehen, wie die Normsetzung sich im 16. Jahrhundert rasch ausbreitet und ansteigt bis in die 80er Jahre des 18. Jahrhunderts. Fast gleichzeitig mit der Französischen Revolution geht diese Art Normsetzung zu Ende. Das Ende ist also klar, aber weniger der Anfang. Aber ich glaube, mit dem Wort „Verdichtung“ kann man es doch angemessen beschreiben. Diese Verdichtung setzt 1495 mit der maximilianischen Reichsgesetzgebung ein, erst recht dann mit den Reichspolizeiordnungen und den Ausstrahlungen auf die Territorien. Und die dritte Bemerkung ist nur eine Vormerkung für die Schlussdiskussion. Herr Starck, wir sollten uns einmal am Ende fragen, ob wir heute wirklich einen liberalen Staat haben. Ich glaube das längst nicht mehr, nämlich einen liberalen Staat in dem Sinne, wie ihn das 19. Jahrhundert imaginiert hat. Wir sind längst im modernen, permanent influenzierenden, pädagogisierenden Staat - insbesondere auf europäischer Ebene - angekommen. 
SELLERT:

Jetzt hat Herr Simon das Wort.

SIMON:

Zunächst zu den Bettelverboten: In der Polizeiliteratur finden sich auch lange Erörterungen über den Sinn und Zweck von Bettelverboten und auch da können Sie ganz deutlich einen Motivwandel erkennen. Zu Beginn der Neuzeit überwiegt das Motiv der Bekämpfung des Müßiggangs, aber nicht aus ökonomischen Motiven, sondern weil Müßiggang aller Laster Anfang ist. Das steht im Vordergrund. Der zweite Punkt ist die Begrenzung der Gemeinschaftslasten für den Unterhalt der Bettler. Denn wenn das Verhältnis zwischen Leistenden und denen, die die Hand aufhalten, außer Balance gerät, stürzt die lokale Gemeinschaft ökonomisch ab. Im 18. Jahrhundert kommt dann der Gesichtspunkt hinzu, dass Bettler unproduktiv seien, weshalb man sie in den Arbeitsprozess eingliedern müsse.

Zur Frage von Herrn Wißmann: Gegensatz oder Übergang, das ist natürlich immer eine Frage der Darstellung. Es ist ja auch angeklungen bei Herrn Starck: Zu Beginn des 19. Jahrhunderts gibt es Stimmen, die noch die erziehende Gesetzgebung befürworten und sich dafür stark machen. Aber wenn Sie eine bestimmte These vermitteln wollen, dann werden Sie stärker die Gegensätze zwischen den Epochen betonen. Dass es bei genauem Hinsehen natürlich fließende Übergänge gibt, ist eigentlich klar. Aber es geht ja darum, dass man die leitenden Prinzipien, die hinter dieser Gesetzgebung stehen, herausarbeitet, und da kommt man nicht darum herum, gewisse Grenzen zu ziehen. Außerdem: Was Sie zu außerrechtlichen Institutionen der Volkserziehung sagen, ist kein Einwand, sondern eine - wie ich finde, plausible - Erklärungsmöglichkeit, warum sich der Staat aus dem Geschäft der Erziehung per Gesetzgebung im 19. Jahrhundert zurückziehen kann: Weil er andere, unter Umständen sogar unmittelbarer wirkende Instrumente der Volkserziehung hat. Was die Gesetzgebung anbelangt, so haben Sie vollkommen Recht: Erst seit dem Naturrecht strebt man ganz bewusst danach, die Normen in den neuen Gesetzbüchern auf hohem Abstraktionsniveau zu formulieren. Die Polizeiordnungen sind davon noch weit entfernt. Das sind alles ganz konkrete, auf bestimmte Lebenssituationen bezogene Normen, die noch außerhalb dieses Strebens nach Abstraktion, wie man es seit dem ausgehenden 18. Jahrhundert zu verzeichnen hat, liegen.

SELLERT:

Jetzt darf ich nacheinander die Herren Avenarius, Heun und Link um ihre Diskussionsbeiträge bitten. 
Avenarius:

Herr Simon, Sie haben sich nicht nur unmittelbar an den Polizeiordnungen orientiert, sondern den Rahmen etwas weiter gespannt. Ich werde mir erlauben, das auch zu tun, und einen Punkt erwähnen, anknüpfend an die Bemerkung, die Bestimmungen, die Sie behandelt haben, dienten in der frühen Neuzeit ausschließlich der Veränderung sozialer Strukturen. Gibt es nicht auch den Fall, dass soziale Strukturen nicht verändert, sondern gerade konserviert werden sollen? Denn im Zusammenhang mit einer Erziehung, die bestimmte Verhaltensmuster bei jemandem etablieren soll, steht doch auch, dass man versucht, bereits bestehende ,gute“ Verhaltensmuster vor schädlichen Einflüssen zu bewahren, und auch das mag ja im Wege von Gesetzen geschehen. Ich denke hier an das Zarenreich, wo man wiederholt erlebt, dass ein Zar, der die Vorstellung hat, die Menschen müssten erzogen werden, versucht, ,schädliche“ Einflüsse fernzuhalten, indem er Auslandsreisen oder Bücher verbietet, Universitäten schließt usw. Das lässt auf ein Menschenbild schließen, in dem das, was Sie skizziert haben, gerade nicht gegeben war: dass man voraussetzen darf, dass die Menschen in der Lage sind, Vorgaben zu befolgen, und auch vernünftigerweise den Willen entwickeln, sich normgerecht zu verhalten. Denn entweder können diejenigen, die diese ,schädlichen“ Anregungen aus dem Westen bekommen, sich danach nicht mehr ,richtig“" verhalten oder sie wollen das dann schlicht und einfach nicht mehr. Ist das eine radikale Ausnahme oder beobachten Sie in dem Bereich, den Sie in erster Linie betrachtet haben, derlei auch?

Sellert:

Ich darf jetzt Herrn Heun bitten.

HEUN:

Ja, entgegen manchen vorherigen Diskutanten habe ich Sie eigentlich gar nicht so verstanden, dass der entscheidende Bruch im 19. Jahrhundert liegt. Da liegt zwar ein Bruch, aber ich habe Sie eigentlich eher so verstanden, dass wir mit Reformation, Konfessionalisierung und Sozialdisziplinierung im 16. und 17. Jahrhundert einen umfassenden Anspruch beobachten und im 18. Jahrhundert schon eine ganz entscheidende Reduktion, die darin liegt, dass der Anspruch auf das Ökonomische reduziert wird. Also, wir haben schon einen Differenzierungsprozess, und insofern ist das auch eine natürliche Entwicklung im 19. Jahrhundert, wenn man nämlich im 18. Jahrhundert sieht, dass die ökonomische Entwicklung, mit АDAm Sмiтн zu sprechen, entscheidend durch die Individuen selbst und die unsichtbare Hand gefördert wird, dann braucht man den Einsatz des Staates nicht und dann kann sich der Staat dort ohne Weiteres herausziehen. Das heißt, die spätere Entwicklung im 19. Jahrhundert ist schon damals angelegt. Der Perspektivenwechsel ist nur das Entscheidende, dass das Kollektive im 
18. Jahrhundert im Vordergrund steht und mit der Theorie von AdAm Smiтh genauso wie mit der Entwicklung von Grundrechten und der Individualisierung alles umgestellt wird auf das Individuum und damit dann natürlich der Staat in eine ganz andere Rolle kommt und sich aus diesem Grund sehr viel stärker zurückziehen kann und auch ganz konsequent sich dann zurückzieht. Nur eine ganz kurze Bemerkung auch noch zu den Entwicklungen davor. Zu den Bettelverboten haben Sie ja schon selbst gesagt, dass das eine andere Zielsetzung war. Ich meine auch die Hochzeitsregelungen und Essverbote usw. haben einen ganz anderen Sinn, nämlich die sollen die Standesgrenzen sehr viel stärker betonen und ziehen. Der Erziehungsgedanke stand also nicht so sehr im Vordergrund.

STOLleis:

Es gibt eine historische Sequenz im Motivwandel: Zuerst ging es darum, die Sünde zu verbieten und dann kommt das Ständische und dann das Ökonomische.

HEUN:

Ja, aber auch gerade das Ständische ist ein Gesichtspunkt, der dann sehr viel stärker zurücktritt, der in den Polizeiordnungen im 16. und 17. Jahrhundert noch da ist, aber dann doch deutlich zurücktritt. Sie haben ja auch kaum auf die Instrumente abgestellt. Die Polizeiordnungen kamen, wie Herr Sellert ja schon bemerkt hat, eigentlich kaum in den Blick, sondern die dahinterliegenden Zielsetzungen. Wenn man über die Instrumente reden würde, dann müsste man über die Entwicklung der Polizeiordnungen, der Erziehung, der Schulen und anderer Einflussinstrumente nachdenken. Aber insofern war ja die Konzentration eigentlich auf die Zielsetzung ein sinnvoller Ansatz angesichts der Kürze der Zeit.

SELLERT:

Herr Link bitte.

LINK:

Herr Heun hat gerade schon etwas angesprochen, was ich fragen wollte, nämlich wie in dieses Schema die ständische Gliederung hineinpasst. Ich ziehe diese Frage zurück. Ich möchte aber vor allem an das anknüpfen, was Herr Starck gesagt hat - an das Problem der Konfessionalisierung. Sie haben darauf schon geantwortet. Ich habe hier nur einige Bedenken, wenn Sie sagen, die katholische Kirche habe in den katholischen Ländern ihre Selbstständigkeit sehr viel wirksamer behauptet als die evangelischen Landeskirchen in den protestantischen Territorien. Kann man das so sagen, wenn man sieht, mit welcher Radikalität etwa JosePH II. in das kirchliche Leben eingreift? Das geht bis hin zur Zahl der Kerzen, die auf dem Altar angezündet werden dürfen, zur Abschaffung eines 
Großteils der kirchlichen Feiertage, zur mit der Pfarrregulierung verbundenen Reform der Seelsorge usw. Lässt sich das alles auf primär wirtschaftliche oder gar auf säkularisierende Intentionen reduzieren? Das ist mir zu einfach. JosEPH II. ging es sicherlich - auch - um einen Reformkatholizismus im Sinne der christlichen - Aufklärung: Nicht der aufwendig praktizierte äußere Kultus ist das Lebensprinzip der Kirche, sondern die innere Frömmigkeit, auf die es allein ankommt. Die josephinischen Reformen lassen sich sicher auch, aber eben nicht allein auf ökonomische Gründe verrechnen.

\section{SIMON:}

Natürlich. Die Frage ist: Wie waren der Josephinismus und das josephinische Staatskirchentum motiviert? Dass hier jedenfalls auch ökonomische Gründe wichtig sind, sieht man etwa an der Reduktion der Feiertage. Das war eine allgegenwärtige Forderung in der kameralistischen Literatur: Feiertage abbauen, weil da nicht gearbeitet wird. Man sieht es auch an der Unterscheidung zwischen den beschaulichen Klöstern und den Seelsorgeorden. Die beschaulichen Klöster, die tun nichts, die betteln nur, die werden abgeschafft, während die Predigerorden bestehen bleiben und dadurch nützlich gemacht werden, dass man sie in die Gemeindeseelsorge stärker integriert. Das Vermögen der beschaulichen Orden wird beschlagnahmt, verstaatlicht und zu nützlichen Bildungszwecken verwendet; das fließt in Schul- und Universitätsfonds usw. Es ist dann eine weitere Frage, inwieweit in diesem Staatskirchentum der Gedanke der Verinnerlichung angelegt ist. Ich bin skeptisch, ob es da wirklich um das Christentum ging; ob das wirklich ein autonomer Gesichtspunkt war. Denn diesen Topos finden Sie ja auch in der kameralistischen Literatur überall; da wird überall gesagt: Kirche und Religion sind wichtig, weil sie die Möglichkeit des unmittelbaren Zugriffs auf die Psyche des Menschen bieten, um seine charakterliche Struktur so zu beeinflussen, dass er sich in die moderne Erwerbsgesellschaft reibungsfrei einfügt. Das findet sich in der kameralistischen Literatur überall und auch im Josephinismus ist das wirksam.

Vielleicht noch eine Bemerkung zu dem Einwand, dass zu wenig über die Polizeigesetzgebung vorgetragen wurde. Wenn Sie die Regelungsgegenstände der Polizeigesetzgebung Revue passieren lassen - das ist ja erfolgt in den Repertorien, die im Max-Planck-Institut für Europäische Rechtsgeschichte in Frankfurt a.M. entstanden sind - dann werden Sie sehen: Die Polizeigesetzgebung folgt getreulich den Grundsätzen, wie sie sich zusammengefasst und konzentriert in der Polizeiliteratur der frühen Neuzeit finden. Es kommt hin$\mathrm{zu}$, dass die frühneuzeitlichen Polizeigesetze, anders als moderne Gesetze, noch ausführlich begründet werden und sich eingehende Rechtfertigungen für diese Gesetzgebungsakte in den Einleitungsteilen finden. Das ist eine Rhetorik, die 
kommt eins zu eins aus den Regimentstraktaten. Die Polizeigesetzgebung ist ein getreulicher legislativer Abdruck dieser Polizeiliteratur. Insofern hätte es keinen großen Sinn gehabt, das als einen eigenständigen Darstellungsstrang aufzubauen. Deswegen der Zugriff auf die Polizeiliteratur, weil man dort in konzentrierter und direkter Form diejenigen Ordnungsvorstellungen geboten bekommt, die hinter dieser Polizeigesetzgebung stehen.

Schließlich noch zum Gesichtspunkt der „Konservierung“, auf den ich hier nicht eingegangen bin. In der Tat versteht sich die Polizeigesetzgebung des 16. Jahrhunderts jedenfalls vom Ansatz her konservierend. Sie will gegebene gesellschaftliche Strukturen bewahren und abschirmen gegenüber dem Wandel. Am deutlichsten sieht man das in der Kleidungsgesetzgebung. Damit sollte die Ständeordnung stabilisiert werden, die durch ökonomische Verschiebungen in Frage gestellt wurde. Auch die Rhetorik der Polizeiordnung ist immer getragen von der Vorstellung: Ursprünglich war es einmal gut, die Dauer der Zeit hat das ursprünglich Gute aber schlecht gemacht und wir wollen zurück zu diesem ursprünglich Guten.

Ohne Zweifel ist die Idee der Polizei im 16. Jahrhundert also noch beherrscht von der Vorstellung ,früher war es besser“ und deswegen hat man die Gemeinschaft wieder zu diesem besseren Ausgangspunkt zurückzuführen. Deswegen ist eben das Stichwort dieser Zeit die „Reformation“, das Zurückführen zu einem vermeintlich besseren Ausgangspunkt. Und das beinhaltet zugleich die Vorstellung: Veränderungen sind eigentlich Verfall. Wenn es bei den ursprünglich besseren Ausgangsbedingungen geblieben wäre, dann wäre es besser. Deswegen die negative Einstellung dieser frühen Politikliteratur zur Veränderung, mutatio ist ein ganz negativ geprägter Begriff.

Das ändert sich im Laufe des 17. und 18. Jahrhunderts. Nunmehr wird die Entwicklungsperspektive in das politische Denken eingeführt. Es ist die Vorstellung, ein Land müsse ökonomisch entwickelt werden. Man entwickelt dabei Perspektiven für die Zukunft, in die hinein das Land bewegt werden soll. Es gibt einen ausgeprägten Perspektivenwandel zwischen den Reformationsvorstellungen im 16. Jahrhundert und den in die Zukunft hineinentwickelten Vorstellungen der Polizeiliteratur des 18. Jahrhunderts. Erstere umfassen auch die Vorstellung, dass man die Gesellschaft, insbesondere Kirche und Schule, frei hält von gefährlichem Ideengut. Und das heißt natürlich: Kontrolle dessen, was gepredigt wird, Kontrolle des Schrifttums usw. Auch hier findet sich wieder dieser bewahrend-konservative Ansatz, den Sie im Zusammenhang mit dem Zarenreich im 19. Jahrhundert erwähnten. 
Sellert:

Ich bitte jetzt um kurze Beiträge und Antworten, weil wir sonst in Zeitnot geraten. Es haben sich gemeldet Frau Scheiwe und Herr Rückert.

SCHEIWE:

Vielen Dank für die Definition und Unterscheidungen und die Darstellungen der Veränderungen. Ich habe mich gefragt, wenn man Erziehung als Vermittlung von Werten und Normen definiert, was ich völlig einleuchtend finde, ob man den Titel des Symposions, „Das erziehende Gesetz“, vielleicht so nennen sollte: „Vom erziehenden Gesetz zum verhaltenssteuernden Gesetz“, um die Vermischung der Begriffe zu vermeiden. Verhaltenssteuerung setzt auf Anreize, positive und negative, aber Erziehung will in irgendeiner Art und Weise Werte vermitteln. Auch der Begriff „Edukationseffekt“, wie er in der Bundesverfassungsgerichtsrechtsprechung verwendet wurde, ist ja nicht ganz klar. Es geht dabei im Grunde genommen nicht um Werte, sondern um Anreize in eine bestimmte Richtung. Diese Unterscheidung würde ich auch aus Ihrer Darstellung der Veränderungen ableiten, wobei es natürlich auch Recht gibt, das Erziehung und Sozialisation regelt und insofern auch immer bestimmte Erziehungsziele umfasste oder Konflikte über Erziehung wie etwa zwischen Kirche und Staat um den Religionsunterricht auslöste. Wäre es daher nicht sinnvoll, zwischen Erziehung und Verhaltenssteuerung zu differenzieren und auch so etwas wie den Begriff „Edukationseffekt“, in dem Motive und Wirkung vermischt werden, zu vermeiden? Würden Sie das auch so sehen?

Sellert:

Herr Rückert bitte.

RÜCKERT:

Ich ziehe Einiges zurück, halte aber noch an zwei Bemerkungen fest. Die eine ist zustimmend - es ist ja alles wunderbar klar gewissermaßen für die Frühe Neuzeit, schöne Texte, schöne Quellen, klare Aspekte, schöne Beispiele. Aber eine Facette ist nicht vorgekommen, die Sie jetzt gerade angeschnitten haben mit dem Wort ,Zukunft“. Und damit meine ich, es gibt eine große Umstellung im Denken, ob man das Ideal nach vorne erstrebt oder nach hinten, konkret gesagt: Die Romantiker sagen natürlich: zurück zum ursprünglichen Zustand der Unschuld und der Freiheit. Und dahin wollen sie erziehen, Rousseau steht dahinter. Ihre Texte dagegen sagen alle: Nach vorne, zur perfekten Moral einerseits oder zur perfekten Ökonomie andererseits. Das finde ich interessant und das führt mich auf eine zweite Bemerkung, die daran anschließt. Also außer ADAM Sмітн, Herr Heun, natürlich mit seiner Individualperspektive, muss man, glaube ich auch, ein bisschen beachten oder als Indikator für Umbrüche verwenden, 
was die Pädagogik macht, denn diese kümmert sich um die Erziehung und es gibt einen ganz klaren Bruch zur Individualerziehung im späten 18. Jahrhundert. Das ist vorher überhaupt kein Thema, Goethe schreibt den Wilhelm Meister, da geht es nur um Erziehung, um Ausbildung des Individuums Wilhelm und und und, könnte man aufzählen. Das finde ich schon wichtig, wenn man nachdenkt über Brüche und Motive und womöglich auch noch über Kausalitäten.

SELLERT:

Ich knüpfe an das an, was Herr Stolleis gesagt hat. Polizeiordnungen enthalten eine Fülle von Regeln, aus denen sich kraft ihrer Gestaltung der Erziehungsgedanke ergibt: Kleiderordnung, Trinkordnung usw. Aber es muss doch zunächst einmal die Frage gestellt werden, welche Polizeiordnungen wir überhaupt meinen. So gibt es die Reichspolizeiordnungen von 1530, 1548 und 1577, die möglicherweise eine etwas andere Funktion als die zahlreichen territorialen Polizeiordnungen haben. In den Reichspolizeiordnungen geht es jedenfalls ganz entscheidend darum, den Frieden im Reich zu erhalten. Es gibt einen bekannten Prozess gegen den Hallenser Professor Christian Thomasius. Dieser wurde 1714 am Reichshofrat wegen Verletzung der Reichspolizeiordnung angeklagt, weil er Kirche und Papst durch einen von ihm zu verantwortenden Kupferstich verunglimpft hatte. Wie dieser Fall zeigt, geht es in den Reichspolizeiordnungen in erster Linie nicht um Erziehung, sondern um die Wahrung des Friedens im Reich; im Thomasıus-Fall also hauptsächlich um den Religionsfrieden. Möglicherweise ist stattdessen in den territorialen Polizeiordnungen, auch wenn für diese die Reichspolizeiordnungen Vorbild gewesen sind, der Erziehungsgedanke deutlicher verankert, was auch in der von Ihnen ausgewerteten zeitgenössischen Literatur sichtbar geworden ist.

Also, ich meine, wir müssten hier diese Polizeiordnungen unterscheiden, wenn man nach ihrem Erziehungscharakter fragt. Vielleicht kommt der Erziehungscharakter in den territorialen Polizeiordnungen, die, das weiß ich natürlich, nach den Reichspolizeiordnungen ganz wesentlich gestaltet worden sind, aber doch viel deutlicher zum Ausdruck, insbesondere wenn man dann die Literatur hinzunimmt, die Sie ausgewählt haben.

Meine nächste Frage betrifft die Strafen, die in den Polizeiordnungen vorgesehen sind. Hatten diese Strafen eine Erziehungsfunktion? Das hängt damit zusammen, welchen Zweck die Strafe hatte. Sollten sie Vergeltungs- oder Präventionscharakter haben? Nur im letzten Falle kommt eine Erziehungsfunktion der Strafe in Betracht. Tatsache ist, dass über die Strafzwecke in den von Ihnen behandelten Epochen lebhaft diskutiert wird. Hinzu kommt die sich vollziehende Trennung von Strafjustiz und Policey, womit sich nicht nur die Zuständigkeiten für strafrechtliche Tatbestände änderten, sondern vermutlich auch die 
Strafen unterschiedliche Funktionen erhielten. Parallel zu diesen Entwicklungen gewann die Freiheitsstrafe an Bedeutung - angefangen von dem ersten Londoner Zuchthaus Bridewell um 1550 über die Gründung des Männerzuchthauses in Amsterdam (1595) bis hin zur Errichtung des Zuchthauses Celle zu Beginn des 18. Jahrhunderts -, mit der zusammen der Erziehungsgedanke in den Mittelpunkt rückte. Meine Fragen lauten also: Muss man für den Erziehungsgedanken zwischen den Reichs- und den territorialen Polizeiordnungen unterschieden? Welche Rolle spielen für den Erziehungsgedanken die in den Polizeiordnungen angedrohten Strafen und in diesem Zusammenhang das Verhältnis von Justiz und Polizei?

SIMON:

Ja, das spielt eine riesige Rolle. Zugespitzt könnte man die These folgendermaBen formulieren: Im 19. Jahrhundert wird der Erziehungsgedanke in der Polizeigesetzgebung fragwürdig; in der Verwaltungstätigkeit bedarf er daher zusätzlicher Legitimierung. Er verliert seine überlieferte Selbstverständlichkeit. Er wird fragwürdig vor dem Hintergrund eines liberalen Staatsverständnisses. Gleichzeitig kommt der Erziehungsgedanke nun aber in der Tat im Strafvollzug zur Geltung. Das lässt sich an der utilitaristischen Strafzwecklehre der Aufklärung deutlich ablesen. Bei CARPzov kann man sehen, dass der Strafzweck überwiegend noch in den Kategorien eines religiös unterfütterten Vergeltungsdenkens definiert wird. Der Fürst muss die Verbrecher bestrafen, weil ein unbestraftes Verbrechen die göttlichen Strafen auf das Land zieht. Bei PufEndorf wird dann der Präventionsgedanke sichtbar: Strafe hat also den Zweck, zukünftige Taten zu vermeiden. Noch stärker kommt der Erziehungsgedanke im Strafrecht dann im 19. Jahrhundert mit der Resozialisierungsidee zur Geltung, mit der Vorstellung also, dass man die Freiheitsstrafe dazu nutzt, in einer Art Therapie auf den Charakter und die Mentalität des Verbrechers einzuwirken. Aber eben nur im ,besonderen Gewaltverhältnis“ und nicht allgemein in der Gesellschaft. Im Polizeirecht und im Verwaltungsrecht des 19. Jahrhunderts hingegen wird der Erziehungsgedanke problematisch. Die Frage ist, wie man diese Verlagerung des Erziehungsgedankens von der Polizei weg in den Strafvollzug zu bewerten hat. Denn dazu eignen sich ja nur langfristige Freiheitsstrafen, denn nur sie eröffnen die Möglichkeit, den Verurteilten an Arbeit zu gewöhnen, ihm sozialfreundliches Verhalten einzuimpfen usw.

Zur Frage der Differenzierung zwischen Reichspolizei- und territorialen Polizeiordnungen: Ich glaube, dass man den Dingen besser auf den Grund kommt, wenn man die territorialen Polizeiordnungen betrachtet, weil der Schwerpunkt der Verwaltungstätigkeit im Heiligen Römischen Reich doch mehr und mehr bei den Territorien lag. Das Reich hat ja dann doch letztlich nur noch eine 
Koordinierungsfunktion, während die intensive Verwaltungstätigkeit in die Fläche hinein von den Territorien gemacht wird, und deshalb bildet sich der Wandel der Verwaltungszwecke eben auch viel deutlicher in der territorialen Gesetzgebung ab, die im Übrigen auch von der Masse her seit dem 17. und 18. Jahrhundert die Polizeiordnungen des Reichs um ein Vielfaches übertrifft. Deswegen hat man, wenn man den Verwaltungszwecken nachspürt, hier den Akzent zu setzen.

Schließlich zur Abgrenzung von Erziehung und bloßer Verhaltenssteuerung: Da würde ich Ihnen zustimmen. Das ist, glaube ich, eine ganz grundsätzliche Frage, die sich bei dieser Tagung stellt: Kann man jede Form der Verhaltenssteuerung durch Strafe oder Belohnung als edukatorische Gesetzgebung bezeichnen? Von edukatorischer Gesetzgebung kann man meines Erachtens nur dann sprechen, wenn die Gesetzgebung getragen ist von der Intention des Gesetzgebers, tatsächlich auf die mentalen Strukturen der Normadressaten zugreifen zu wollen. Ansonsten wäre der heftige Affekt der frühliberalen Bewegung gegen den erziehenden Staat kaum erklärbar. Denn dieses Votum, etwa von WELCKER, gegen den erziehenden Staat war ja kein Votum gegen Steuerungsgesetzgebung allgemein. Dagegen hatte man natürlich nichts. Es ist ja keineswegs so, dass Welcker jede Form motivierender Gesetzgebung ausschließen wollte, denn ohne das kommt der moderne Staat ja überhaupt nicht aus. Das war mitgedacht bei Welcker. Es muss hier also aus der Sicht des 19. Jahrhunderts gravierende Unterschiede gegeben haben zwischen allgemeiner Verhaltenssteuerung im Wege der Gesetzgebung und einer eigentlich „erziehenden“ Gesetzgebung mit weiterreichenden Intentionen.

Sellert:

Vielen Dank Herrn Simon und allen Diskutanten. 